\title{
Therapy in Women with Recurrent Miscarriage Depending on Morphological Characteristics of the Endometrium
}

\author{
Solovyeva $\mathrm{AV}^{*}$, Gace $\mathrm{V}$ and Gerasimova $\mathrm{OP}$ \\ ${ }^{1}$ Department of obstetrics and gynecology, University of Russia (RUDN University), Russia \\ ${ }^{2}$ Department of Medical Sciences, Russia
}

Submission: November 24, 2017 ; Published: December 18, 2017

*Corresponding author: Solovyeva AV, Department of obstetrics and gynecology, University of Russia (RUDN University), Russia, Email: av_soloveva@mail.ru

Abstract

Recurrent miscarriage takes one of the first places among obstetrical problems [1-3]. The frequency of spontaneous termination of pregnancy in Russia is quite high from $15 \%$ to $23 \%$ among all registered pregnancies. About $80 \%$ of reproductive losses occur in the first trimester. In obstetrical practice in Russia the notion of habitual or recurrent loss of pregnancy is widespread and means abortion 2 or more times in a row in one woman. After 2 interruptions $88 \%$ of women show significant functional and structural changes in the reproductive system, which makes it possible to verify diagnoses that do not presuppose self-healing. This is confirmed by the every year increasing appeal to clinics of women with repeated pregnancy losses.

We also share the opinion of [2,3] it is possible to establish the cause of repeated pregnancy losses in $50 \%$ or more couples, and in $2 / 3$ of the pairs can achieve a successful outcome. The habitual loss of pregnancy has many factors: anatomical, autoimmune, genetic, inflammatory, etc. [2-4]. Qualified pathomorphological examination of aspirates from the cavity uterus can help to identify the cause of "fading" of pregnancy and the solution of diagnostic problems and therapeutic measures in a woman [5].

Keyword: Recurrent miscarriage; Extragenital diseases; Repeated pregnancy losses

\section{Introduction}

We examined 118 women with habitual loss of pregnancy which were divided into two groups. The first (main) group included 78 women who applied to the clinic to get rid from the undeveloped pregnancy and had a history of pregnancy with abortive outcome, stillbirth, etc. The second group (control group) included 40 women with the same diagnosis, observed in municipal women's consultations. Women were selected empirically for comparative analysis.

\section{Materials and Methods}

The pathomorphological study of aspirate in women of the first (main) group was performed in the Research Institute of Human Morphology of the Russian Academy of Medical Sciences (head of the laboratory of the pathology of the female reproductive system, Corresponding Member of the Russian Academy of Natural Sciences, Professor Milovanov A.P.). The material obtained by vacuum aspiration was rinsed from excess blood and fixed in a $10 \%$ formalin solution. The material was treated according to the scheme: it was filled in paraffin sections were prepared and then stained with hematoxylin and eosin.
We looked through the micro-preparations according to the algorithm:

a. The fragments of parietal endometrium.

b. Utero-placental area zone of direct contact of placental anchor villi and maternal decidual membrane of the uterus.

c. Separately examined the wall of the chorionic sac and the villi (floating) outgoing it.

\section{Results}

If in the studied material predominated the characteristic signs of acute abscess or chronic endometritis then in that case the dominant cause was "Inflammation" (Figure $1 \& 2$ ). A distinctive feature of acute abscessed endometritis from chronic was the presence of a cluster of segmented leukocytes and local small lymphocytes on a large area of the endometrium, as well as the spread of inflammatory cells between the rows of decidual cells of intermediate and epithelioid types, plethora of the vessels with loss of the complete contour of endothelial cells. In chronic endometrium in parietal endometrium and utero-placental area 


\section{Journal of Gynecology and Women's Health}

predominated maternal lymphocytes, plasma cells and only a small part of segmented leukocytes. If in the studied material predominated the signs of weak or insufficient decidualization of the stroma of parietal endometrium and utero-placental area which is a sign of hormonal disturbances, then the dominant cause was luteal phase deficiency (Figure 3).

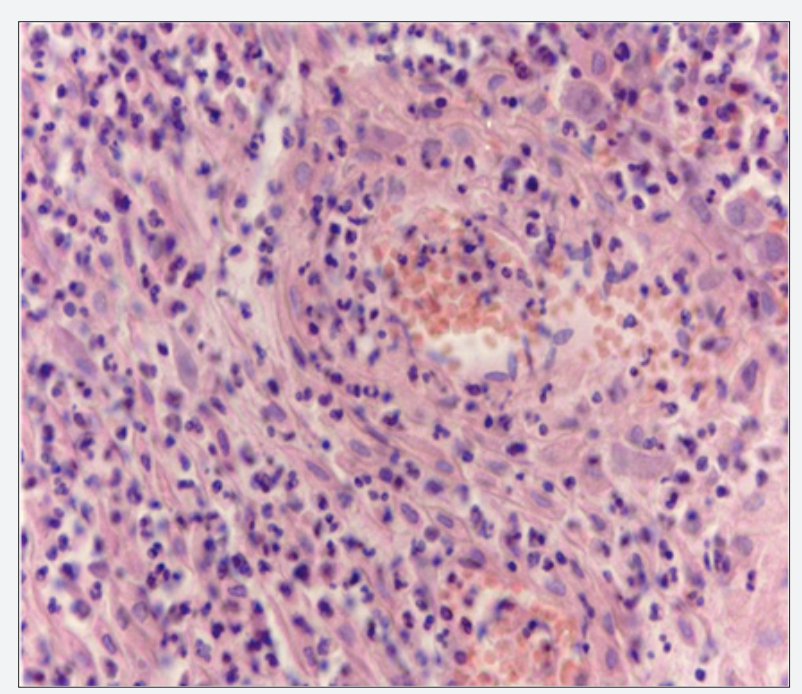

Figure 1: Acute abscessed endometritis. Area of distribution of segmented leukocytes among decidual cells. Stained with hematoxylin and eosin $\times 100$.

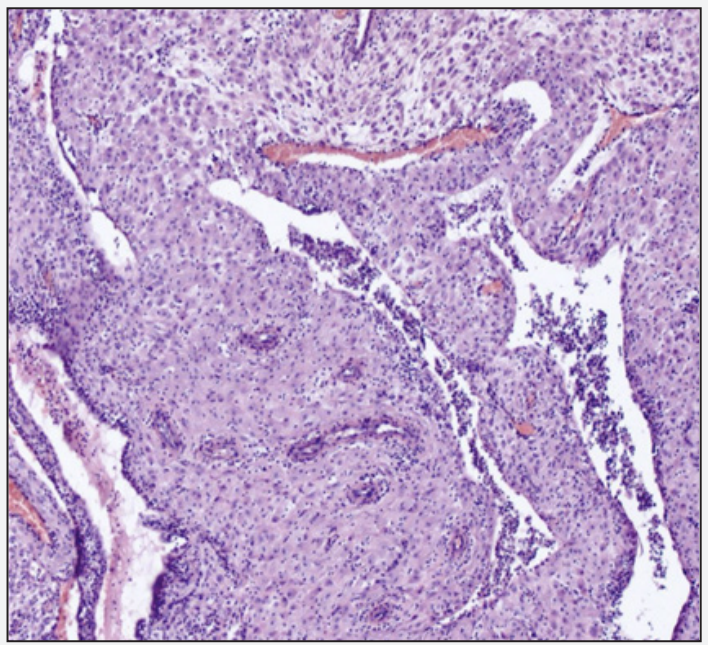

Figure 2: Chronic endometritis. Primary lesion of glands with penetration of infiltrate into their lumens. Vasculitis staining with hematoxylin and eosin $\times 400$.

When in the material were detected a massive thrombosis of micro vessels in the parietal endometrium and retrochorial hematoma the dominant cause was "thrombohemorrhagic disorders" (Figure 4). In women of the control group, scraping from the uterus was assessed in municipal laboratories. We collected and analyzed anamnestic data of patients in both groups: marital status; age; education; the formation of the reproductive system of adolescent girls; beginning of sexual activity; social and living conditions; presence of bad habits; occupational hazards, presence of somatic diseases, autoimmune diseases; and obstetric-gynecological anamnesis: gynecological diseases; surgical interventions; the number and timing of interruption of previous pregnancies, as well as their course.

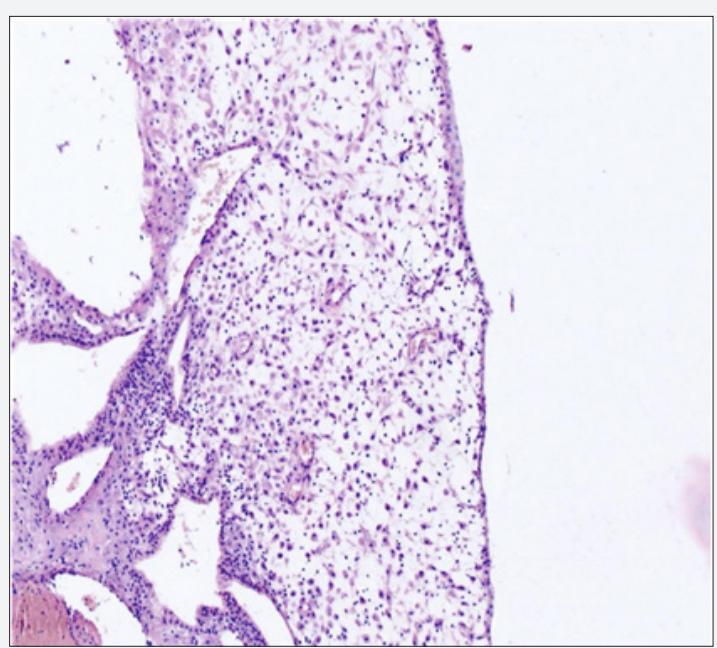

Figure 3: Weak decidualization of the stroma of the parietal endometrium with progesterone deficiency. Rare location of stromal cells, elongated glands with epithelial atrophy and scant secretion Stained with hematoxylin and eosin $\times 400$.

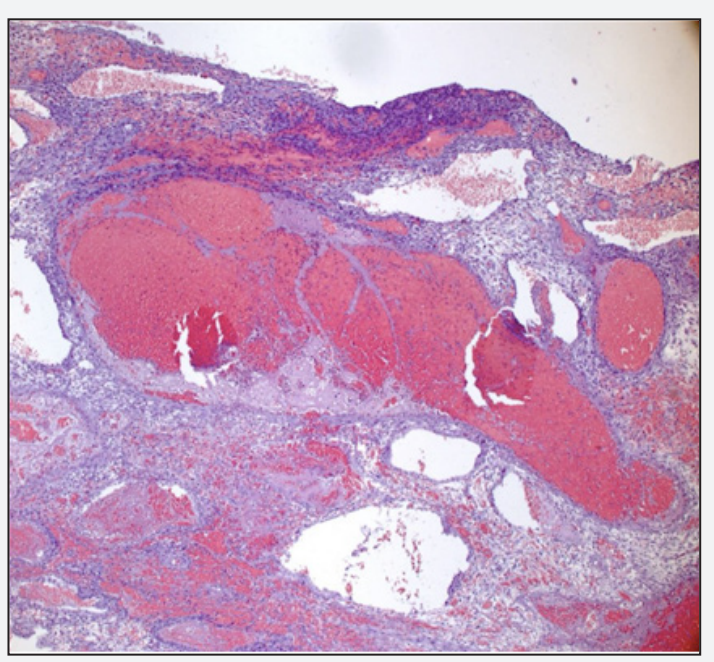

Figure 4: Hemorheological abnormalities as the cause of undeveloped pregnancy in the 6th week of pregnancy ("old thrombus" in the vessel of the parietal endometrium). Staining with hematoxylin and eosin $\times 50$.

The age of the examined patients in both groups ranged from 18 to 42 years which corresponds to a favorable reproductive period. According to the marital status at the time of gestation women in both groups were in most cases married: 75 (96.1\%) in the main group and 35 (87.5\%) in the comparison one.

When analyzing the structure of extragenital morbidity, there were significantly more diseases in women with a dominant cause of "thrombohemorrhagic disorders" an average of 2.7 diseases per woman. Compared with women with luteal phase deficiency and "inflammation" (2.1 and 1.9, respectively). They 
also had 2 times more frequent diseases of the cardiovascular system, urinary system, nonspecific chronic lung diseases. Gynecological morbidity in the main group was 1.3 times higher than in the control group. Diagnosis of chronic endometritis, salpingooophoritis, endometriosis, secondary infertility in the main group occurred twice as often as in the control group. Surgical interventions on the uterus and appendages were performed for every $5^{\text {th }}$ woman in the control group and in the main group for every third woman. This was confirmed by the morphological study: almost half of the women from the "inflammation" subgroup (42.9\%) had a history of surgical interventions.

Hormonal disorders mainly in the form of hyperandrogenism were practically identical in both groups and the results were quite high: $32.5 \%$ in the main group and $35.9 \%$ in the control group. In both groups there was a high frequency of artificial termination (abortion) of the first pregnancy: 26.9\% in the main group and $27.5 \%$ in the control group. Vaginal biocenosis was examined in all patients of the main groups (Figure 5) by bacterioscopy, PCR diagnostics and virological research. Chlamydia infection and trichomoniasis were detected only in the subgroup "inflammation" $3.2 \%$ and $6.7 \%$, respectively. It should be noted that all examined women in the "inflammation" subgroup had no monoinfection and a mixed viral-bacterial infection was diagnosed. Attention is drawn to the widespread prevalence of HSV, CMV and HPV. It is not known whether they play a causal role in the recurrent miscarriage.

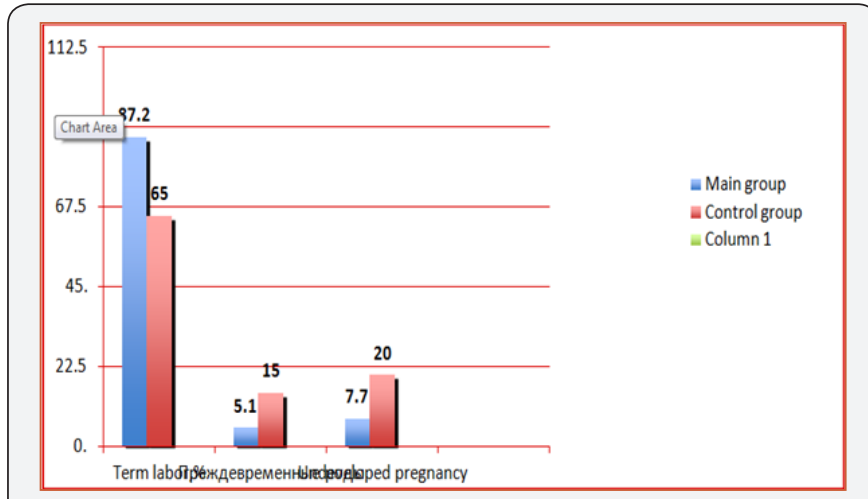

Figure 5: Outcomes of pregnancies in observation groups.

In our study in 28 women with "inflammatory endometrial reaction" the therapy included:

a. Elimination of the pathogen - antibacterial therapy (broad-spectrum antibiotics) taking into account the sensitivity of the infection in combination with metronidazole.

b. Restoration of intestinal microflora and vaginal biocenosis using bifidobacili and lactobacilli.

c. Ozonotherapy for activation of oxygen-dependent processes, regulation of immunity, enhancement of metabolic processes. Local (vaginal) application of high ozone concentrations provided bactericidal, fungicidal and virosocidal effects were against gram-positive, gramnegative bacteria, viruses, fungi and a number of pathogenic protozoa.

d. Restoration of the receptor apparatus of the uterus with the use of physiotherapeutic procedures (ultrasound, magnetotherapy, etc.).

e. Women with chronic salpingooophoritis, endometritis and adhesive process in the small pelvis underwent sanatorium-and-spa treatment.

The effect of the treatment was assessed by the absence of signs of inflammation in the endometrium (Pipel biopsy, morphological conclusion), data of ultrasound investigation (thickness of the endometrium, homogeneous structure, presence of a yellow body in the ovary with a diameter of 16 to $22 \mathrm{~mm}$, presence of blood flow in all layers of the myometrium and blood supply of yellow body). Only in $50 \%$ of patients we received a positive effect of the therapy on all the parameters indicated above in 3 months. In 14 women, according to the results of the morphological study, there was no evidence of inflammation but was observed luteal phase deficiency or insufficient recovery of the endometrial receptors. These patients were administrated with dydrogesterone $20 \mathrm{mg}$ per day from $16^{\text {th }}$ to $25^{\text {th }}$ days of menstrual cycle or micronized progesterone 100-200mg intravaginally for 3-6 menstrual cycles. Pregravid preparation of women with morphological diagnosis of luteal phase deficiency included the regulation of hormonal status. In women with mixed hyperandrogenemia was administered dexamethasone $0.25-0.5 \mathrm{mg}$ at night on the 16 th to $25^{\text {th }}$ day of the cycle for 3 cycles, as well as dydrogesterone $10 \mathrm{mg}$ twice daily or micronized progesterone $100-200 \mathrm{mg}$ intravaginally. In case of decrease in the level of steroid hormones (estrogens) were prescribed drugs of hormone replacement therapy. Estrogens in the form of a gel were applied to the skin $1-2 \mathrm{~g}$ from the $5^{\text {th }}$ to $20^{\text {th }}$ day of the cycle and progesterone preparations from the $16^{\text {th }}$ to the $25^{\text {th }}$ day of the cycle [6].

Treatment was prescribed for 2-3 cycles depending on the reaction of endometrium and ovaries, the parameters of which were controlled by ultrasound. Patients with metabolic disorders pregravid preparation began with the advice of a nutritionist, the selection of a diet aimed at reducing body weight and then the hormonal background began to regulate. When hyperprolactinaemia was detected the drug of choice was cabergoline $0.5 \mathrm{mg} 1$ or $1 / 2$ tablet 1 - 2 times a week depending on the initial prolactin levels and prolactin level control every 2-3 weeks. When hypothyroidism was prescribed eutiroks or L-thyroxine and the dosage was selected individually. The effect of treatment in this group was obtained in $68.6 \%$ of women among them $2 / 3$ women with two or more pregnancy losses in history. Morphological signs of the positive effect of treatment were characterized by the correspondence of endometrium to the late-secretory stage of menstrual cycle. 
In $31.4 \%$ of women who did not have a full-fledged second phase of menstrual cycle. So in addition to progesterone preparations was prescribed physiotherapy to enhance the work of receptor apparatus of the uterus and from the 6th day of the menstrual cycle 10-12 sessions of magnetotherapy. All women had a positive effect of additional treatment. Among women with thrombohemorrhagic disorders there were patients who had a history of antenatal fetal death at term (13.3\%), 13.3\% and $26.7 \%$ of them had three and four pregnancy losses respectively. All patients were examined for antiphospholipid syndrome. Patients who had changes in the coagulative status as well as with a burdened obstetric anamnesis (antenatal fetal death, two or more pregnancy losses) were consulted by a hematologist.

To control the parameters of hemostasis were administrated low-molecular heparins (prophylactic dose). If there was an alteration of aggregation activity of platelets were added anti aggregates (aspirin or dipyridamole). In all cases therapy was supplemented with prescription of progesterone drugs in the II phase of the menstrual cycle for 3-6 months. All patients were administrated within 3-6 months with folic acid 400-800mcg per day. In $92.3 \%$ of the women in the main group after the therapy the pregnancy ended successfully and they gave birth to live full-term children. In $7.7 \%$ of patients again the pregnancy ended unfavorably (5 - undeveloped pregnancy at 6-7 weeks, 1 spontaneous abortion at 21 weeks).

In comparison with the control group, the perinatal outcomes were significantly better (Figure 5), where only $65 \%$ of women had urgent deliveries, $15 \%$ had premature birth. At every fifth women pregnancy stopped in development for up to 20 weeks.

\section{Discussion}

Summarizing the foregoing it should be noted that a differentiated approach to the therapy of women with habitual miscarriage based on a morphological study of the endometrium after the termination of pregnancy and during treatment allows the improvement of pregnancy outcomes (delivery in time) for this contingent of women by $22.2 \%$, and in individual cases by $27.9 \%$. Individual differential treatment of women contributed to a favorable outcome of pregnancy in 85 women with recurrent miscarriage. Thus, in the main group in comparison with the control group, we managed to increase the number of urgent deliveries by almost 1.5 times, reduce the incidence of preterm labor 3 times and 2.6 times the number of undeveloped pregnancies.

\section{Our study showed:}

a. For all known morphological reasons for an undeveloped pregnancy you can select the appropriate corrective treatment.

b. The use of a triad of tests (Pipel biopsy of the endometrium, determination of the level of progesterone in the peripheral blood, ultrasound of the pelvic organs) makes it possible to reliably verify the quality of the treatment, the readiness of the woman for pregnancy and, as a result, to increase the proportion of favorable pregnancy outcomes to 92.3\%.

\section{Conclusion}

A habitual miscarriage is a worldwide and unsolved problem of reproduction [2]. One of the diagnostic methods can be the analysis of structure of endometrium at the termination of pregnancy (vacuum aspiration). Allocation of the dominant cause allows us to narrow down the search for diagnostic tests and choose therapies individually for each woman.

\section{Acknowledgement}

Professor Radzinskiy V.E. Head of the Department of Obstetrics and Gynecology with a course of perinatologyof Federal State Autonomous Educational Institution of Higher Professional Education Russian People Friendship University (Medical Institute) of the Ministry of Education and Science of the Russian Federation, Corresponding Member of Russian Academy of Sciences.

\section{References}

1. Donckers J, Scholten RR, Oyen WJ, Hopman MT, Lotgering FK, et al. (2012) Unexplained first trimester recurrent pregnancy loss and low venous reserves. Hum Reprod 27(9): 2613-2618.

2. Radzinskiy VE (2016) Undevelopied pregnancy. ( $2^{\text {nd }}$ edn.), Revised and additional. GEOTAR-Media, Moscow, Russia, p. 176.

3. Karpa GJ (2017) Recurrent miscarriage: causes, versions and contrasts, treatment. In: Radzinskiy VE (Ed.), Translated from English, GEOTAR-Media, Moscow, Russia, p. 592

4. Jaslow CR, Kutteh WH (2013) Effect of a prior birth and miscarriage frequency on the prevalence of acquired and congenital uterine anomalies in women with recurrent miscarriage: A crosssectional study. Fertil Steril 99(7): 1916-1922.

5. Milovanov AP (2011) Causes and differential treatment of early miscarriage: a guide for doctors. In: Milovanova AP, Serova OF (Eds.), Production and Publishing Complex VINITI, p. 214.

6. Howard JAC (2014) Recurrent Pregnancy Loss: Causes, Controversies and treatment. ( $2^{\text {nd }}$ edn.), CRC Press, USA, p. 456. 


\section{Your next submission with Juniper Publishers} will reach you the below assets

- Quality Editorial service

- Swift Peer Review

- Reprints availability

- E-prints Service

- Manuscript Podcast for convenient understanding

- Global attainment for your research

- Manuscript accessibility in different formats

( Pdf, E-pub, Full Text, Audio)

- Unceasing customer service

Track the below URL for one-step submission https://juniperpublishers.com/online-submission.php 